\title{
Partial least square regression applied to the QTLMAS 2010 dataset
}

\author{
Albart Coster ${ }^{1 *}$, Mario P L Calus $^{2}$ \\ From 14th QTL-MAS Workshop \\ Poznan, Poland. 17-18 May 2010
}

\begin{abstract}
Background: Partial least square regression (PLSR) was used to analyze the data of the QTLMAS 2010 workshop to identify genomic regions affecting either one of the two traits and to estimate breeding values. PLSR was appropriate for these data because it enabled to simultaneously fit several traits to the markers.

Results: A preliminary analysis showed phenotypic and genetic correlations between the two traits. Consequently, the data were analyzed jointly in a PLSR model for each chromosome independently. Regression coefficients for the markers were used to calculate the variance of each marker and inference of quantitative trait loci (QTL) was based on local maxima of a smoothed line traced through these variances. In this way, 25 QTL for the continuous trait and 22 for the discrete trait were found. There was evidence for pleiotropic QTL on chromosome 1. The 2000 most important markers were fitted in a second PLSR model to calculate breeding values of the individuals. The accuracies of these estimated breeding values ranged between 0.56 and 0.92 .
\end{abstract}

Conclusions: Results showed the viability of PLSR for QTL analysis and estimating breeding values using markers.

\section{Background}

Detection of genomic regions affecting traits is a goal in many genetic studies. Studies applying distinct methods for detection of these regions, called quantitative trait loci (QTL), have been described, ranging from single marker regression [1] to methods that enable to fit several markers simultaneously $[2,3]$. Simultaneously fitting all markers leads to more accurate detection of QTL compared to independent fitting of single markers in a regression model when there is linkage disequilibrium (LD) between the genomic regions that affect the trait but comes at the cost of increased computational requirements [2].

Partial least square regression (PLSR) is one method for simultaneously fitting multiple markers and was applied by Bjornstad et al. for detection of QTL [3]. An interesting characteristic of PLSR its straightforward

\footnotetext{
* Correspondence: albart.coster@wur.nl

'Animal Breeding and Genomics Centre, Wageningen University,

Wageningen, The Netherlands

Full list of author information is available at the end of the article
}

application of to simultaneous analysis of data of multiple traits [3].

The objectives of this study were to use PLSR to search for QTL and to estimate breeding values in the dataset of the QTLMAS 2010 workshop.

\section{Material and methods}

Initial analyses

The data were analyzed to identify generation and gender effects. Furthermore, a bivariate animal model was fitted in ASREML [4] using the matrix of additive genetic relations as relative covariance matrix to estimate variance components.

\section{Marker based analyses}

Let $\mathbf{X}$ be the genotype matrix; the number of rows is the number of individuals and the number of columns is the number of markers. Elements of $\mathbf{X}$ are 0,1 , or 2, according to the number of one of the two alleles for that marker in that individual. Let $\mathbf{Y}$ be the matrix of phenotypes; the number of rows is the number of
C Biomed Central

(c) 2011 Coster and Calus; licensee BioMed Central Ltd. This is an open access article distributed under the terms of the Creative Commons Attribution License (http://creativecommons.org/licenses/by/2.0), which permits unrestricted use, distribution, and reproduction in any medium, provided the original work is properly cited. 
individuals and the number of columns is the number of traits in the data (two in these data).

We describe our regression methods in the following sections, beginning with an analysis where each trait was regressed on each marker independently and continuing with PLSR.

\section{Single marker regression}

We used function $\mathrm{lm}$ of $\mathrm{R}$ [5] to regress each trait on each marker. In this analysis, we used the same model for both traits and ignored the non-normal distribution of the discrete trait. We fitted the following model to the data:

$$
\begin{aligned}
& \mathbf{y}_{t}=\mu+\mathbf{x}_{m} b+\mathbf{e} ; \\
& \mathbf{e} \sim N\left(0, \mathbf{I} \sigma^{2}\right) ;
\end{aligned}
$$

where $\mathbf{y}_{t}$ is the vector of phenotypes for trait $\mathrm{t}, \mu$ is a mean, $\mathbf{x}_{m}$ is the vector of genotypes corresponding to marker $\mathrm{m}, \mathrm{b}$ is the unknown regression coefficient of $\mathbf{y}_{t}$ on $\mathbf{x}_{m}$ and $\mathbf{e}$ is the vector of residuals. Inference was based on ANOVA applied to the fitted models.

\section{Partial least square regression}

In PLSR, matrices $\mathbf{X}$ and $\mathbf{Y}$ are decomposed into principal components and loadings:

$$
\begin{aligned}
& \mathbf{X}=\mathbf{T W}^{\prime} \\
& \mathbf{Y}=\mathbf{U Q}^{\prime}
\end{aligned}
$$

where $\mathbf{T}$ and $\mathbf{U}$ are the matrices of scores and $\mathbf{W}$ and $\mathbf{Q}$ are the matrices of loadings [6]. PLSR places two conditions in the decomposition of $\mathbf{X}$ and $\mathbf{Y}$. The first requires orthogonality of $\mathbf{W}$ and $\mathbf{Q}$ and the second requires maximal correlation between the columns of $\mathbf{T}$ and $\mathbf{U}[6]$. After decomposition, $\mathbf{U}$ is regressed on $\mathbf{T}$ :

$\mathbf{U}=\mathbf{T B}+\mathbf{E},(3)$

where $\mathbf{B}$ is an unknown matrix of regression coefficients and $\mathbf{E}$ is a matrix of residuals. We used matrix $\mathbf{B}$ to calculate the matrix of regression coefficients of the individual markers, $\hat{\mathbf{B}}_{m}$ :

$$
\hat{\mathbf{B}}_{m}=\mathbf{T B Q}^{\prime} .
$$

Fitting We treated the data of both traits equally, without accounting for the non-normal nature of the discrete trait. Since PLSR using all marker loci $(\sim 10000)$ was impossible, we calculated the regression coefficients in two steps. First, we regressed the phenotype data on the markers in each chromosome using PLSR and obtained the empirical distributions of these regression coefficients by bootstrapping. Second, we selected the 1000 most significant markers for each trait, defining significance of a marker as the absolute value of its regression coefficient divided by its empirical standard error. Subsequently, we regressed the phenotype data on the selected markers using PLSR and recalculated their standard errors using bootstrapping.

We used the R-package pls [7] to fit, cross validate, and use the PLSR models.

Detecting QTL Our method assumed that the variance explained by markers reaches a maximum in the neighborhood of a QTL. We used locally weighted regression [8] to estimate a smoothed curve through the standardized regression coefficients of the markers, calculated as $\frac{\widehat{\beta}}{\widehat{s e}}$ ( $\widehat{\beta}$ is the estimated regression coefficient for that marker and $\widehat{s e}$ is its empirical standard error, obtained from bootstrapping). We calculated the first and second derivative of this smoothed curve to find local maxima of the curve and we considered these local maxima as QTL. We calculated the variance explained by each QTL as $\sigma_{m}^{2}=2 p(1-p) \widehat{\beta}^{2}$, where $\mathrm{p}$ is its MAF and $\widehat{\beta}$ is its regression coefficient from in the single marker regression analyses.

Calculating $\boldsymbol{E} \boldsymbol{B} \boldsymbol{V}$ We estimated breeding values for all individuals in the data using the regression coefficients for the markers in the second PLSR model. Estimated breeding values (EBV) were calculated as $\mathbf{E B V}=\mathbf{X} \hat{\mathbf{B}}_{m}$.

\section{Results}

Initial analyses

The initial analysis revealed a positive correlation between the traits. No signals of selection nor sex effects were detected in the data.

The results showed that both traits were heritable and genetically correlated. Heritability of the first trait was 0.53 (s.e. 0.06 ) and heritability of the second trait was 0.22 (s.e. 0.04). The phenotypic correlation was 0.25 (s.e. 0.03 ) and the genetic correlation was 0.66 (s.e. 0.09).

\section{Single marker regression}

Figure 1 shows the smoothed curve of the negative logarithm of the significances in the single marker analyses. QTL for the continuous trait were located on chromosomes 1 and 3 with smaller QTL on all chromosomes. The effects of QTL for the discrete trait were smaller compared to the the continuous trait with QTL on chromosomes 1, 2 and 3. Figure 1 suggests at least three pleiotropic QTL; one at approximately half the length of chromosome 1 , one at the beginning of chromosome 3 and another at approximately 0.25 the length of chromosome 4.

\section{Partial least square regression with bootstrapping}

Figure 2 shows the smoothed curves through standardized regression coefficients of the PLSR models. 


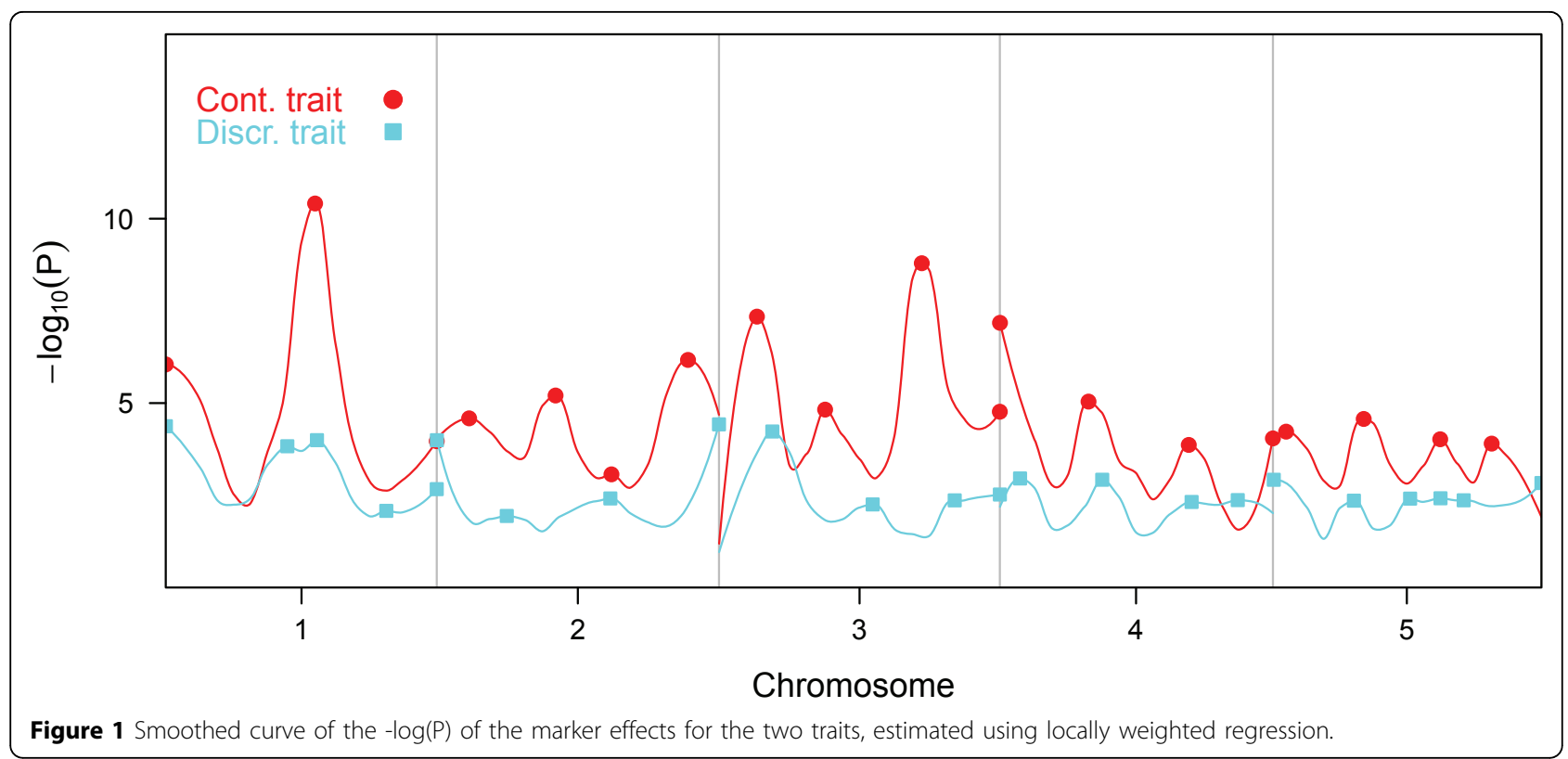

Important QTL for the continuous trait were located on chromosomes 1, 3 and 4. The curves of chromosome 2 were remarkably flat compared to the results in Figure 1. QTL with an important effect on both traits were located on chromosomes 1, 3 and 4 .

Local maxima identified in Figure 2 identified QTL, which are reported in Table 1 with regression coefficients and variance. The variance of QTL, expressed as a proportion of the total genetic variance, was small for both traits. The largest QTL for the continuous trait was located at position 1058 of chromosome 1 and explained $6.8 \%$ of the genetic variance, the largest QTL for the discrete trait was located at position 1977 of chromosome 2 and explained $4.3 \%$ of the genetic variance (Table 1). Based on this table, pleiotropic QTL were located between positions 156 and 159 and positions 494 and 495 on chromosome 1 , between position 3242 and 3274 on chromosome 2, and between position 8890 and 8920 on chromosome 5 because these intervals harbored QTL affecting both traits (Table 1).

The correlations between the EBV and the phenotypes and between the EBV and the true breeding values

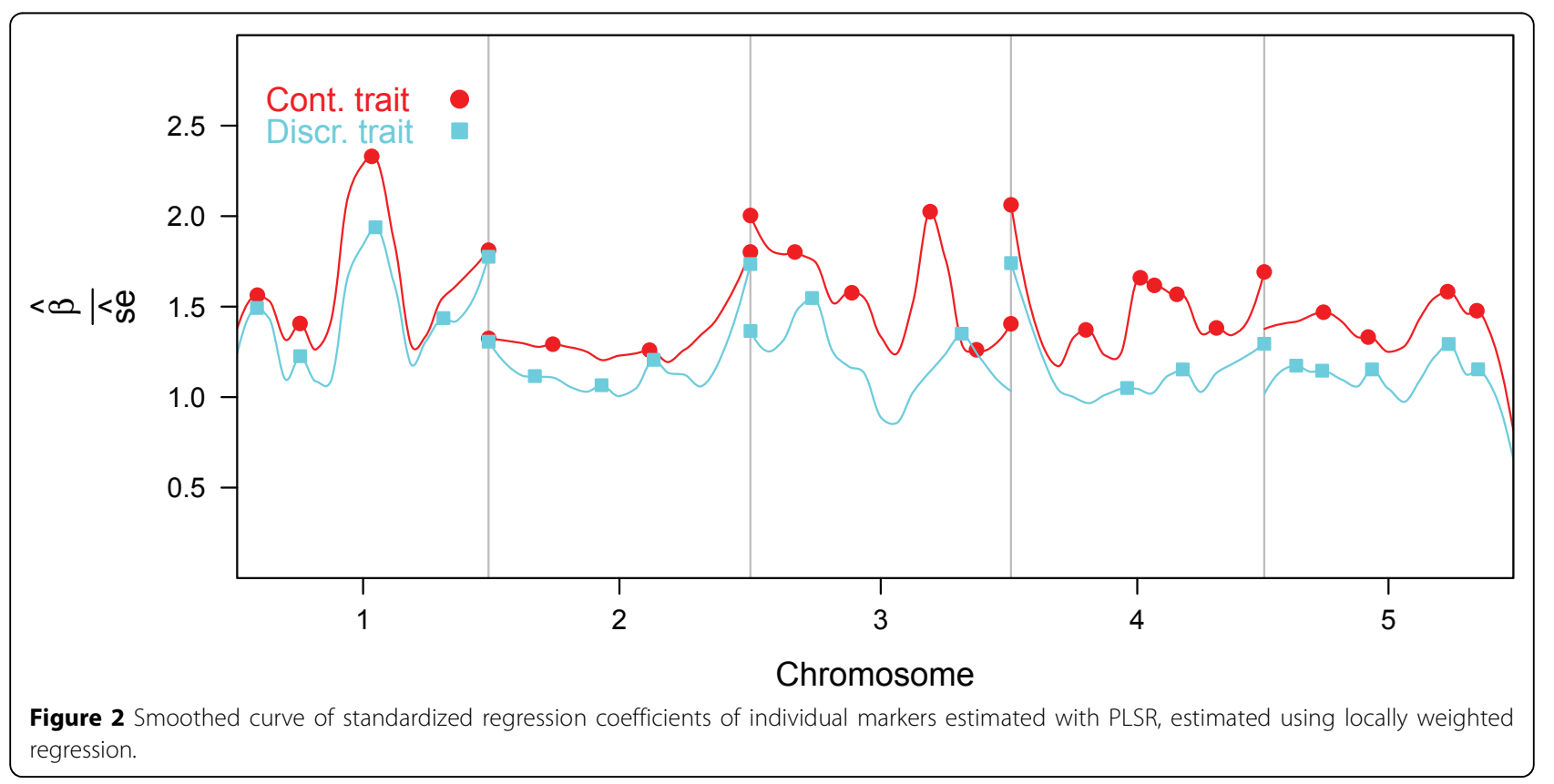


Table 1 Estimated regression coefficients and approximate standard error for the most significant markers in of the second PLSR model. The highlighted cell contain a regression coefficient which was considered most significant, the other cells contain the less significant regression coefficients.

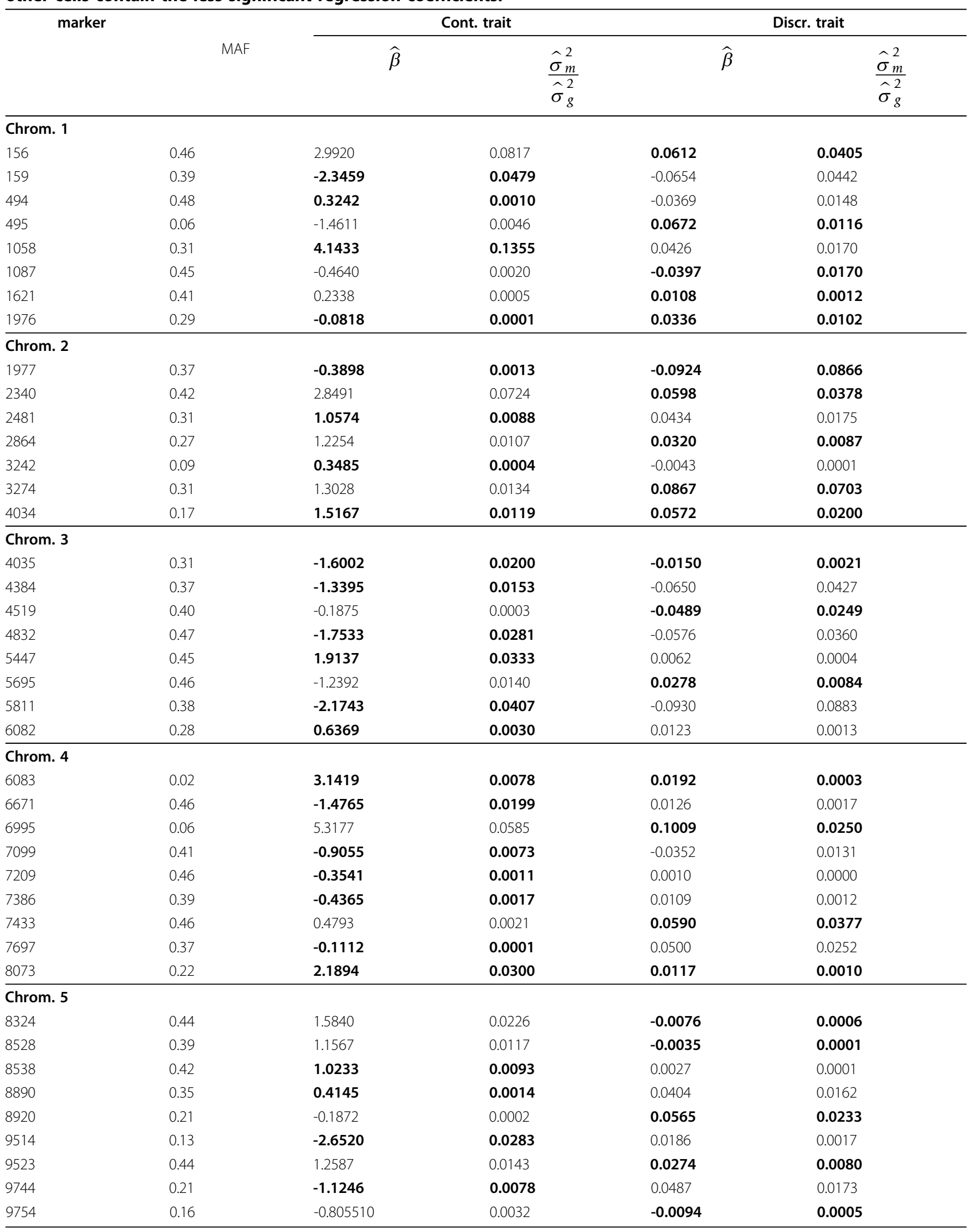


Table 2 Correlations between estimated breeding values (EBV) and phenotypes (P) or true breeding values (TBV) of the continuous and discrete trait of individuals in simulated generations zero to three. TBV of all individuals and phenotypes of individuals in generation 4 were only used to evaluate the correlations but were not used to fit the models

\begin{tabular}{lccccc}
\hline Trait & 0 & 1 & 2 & 3 & 4 \\
\hline Cont. trait & & & & & \\
$r(P, E B V)$ & 0.70 & 0.70 & 0.68 & 0.69 & $\mathbf{0 . 5 2}$ \\
$r(T B V, E B V)$ & 0.79 & 0.73 & 0.66 & 0.69 & $\mathbf{0 . 5 6}$ \\
\hline Discr. trait & & & & & \\
$r(P, E B V)$ & 0.64 & 0.63 & 0.55 & 0.55 & $\mathbf{0 . 3 7}$ \\
$r(T B V, E B V)$ & 0.92 & 0.90 & 0.82 & 0.79 & $\mathbf{0 . 7 2}$ \\
\hline
\end{tabular}

(accuracies of EBV) are displayed in Table 2. All correlations decreased from generation 0 to generation 3 for unknown reasons. The correlations in generation 4 where lower than in the previous generations because these individuals were not used to fit the model. The correlations between the phenotypes and the EBV where highest in the continuous trait but the correlations between the true breeding values and the EBV were highest in the discrete trait (Table 2).

\section{Discussion and conclusions}

We used a non conventional method to infer QTL, based on finding local maxima of a smoothed curves traced through the QTL probabilities (in the single marker regression) and through the standardized regression coefficients (in PLSR). This method assumed that a QTL will appear as a local maximum in the smoothed curves. An advantage of this method over methods that concentrate on profiles of single markers is that it combines evidence provided by a series of markers in the proximity of QTL. A disadvantage is that it does not provide a quantitative test statistic to statistically test for the presence of QTL.

Comparing the QTL detected with our method to true QTL locations revealed differences and similarities. We detected QTL on chromosome 5 while no QTL were simulated on this chromosome. This false detection is inherent to our method since detection was only based on local maxima in the curves. The method suggested many pleiotropic QTL and agreed with the truth, because the majority of the QTL were pleotropic.

We used single marker regression to estimate the variance of individual QTL because we expected that PLSR would underestimate the regression coefficients of QTL in LD with many markers. A disadvantage of this could be biased regression coefficients of QTL in LD with other QTL [2].
The correlations between EBV and true breeding values of individuals in generations 0 to 3 agreed with the correlations of EBV calculated in the studies of Meuwissen et al. [2]. Avoiding the need to preselect markers might lead to higher correlations for the nongenotyped individuals and the method Chun and Keles [9] might be an interesting alternative.

\section{Acknowledgements \\ This article has been published as part of BMC Proceedings Volume 5 Supplement 3, 2011: Proceedings of the 14th QTL-MAS Workshop. The full contents of the supplement are available online at http://www. biomedcentral.com/1753-6561/5?issue=S3. \\ Author details \\ 'Animal Breeding and Genomics Centre, Wageningen University, Wageningen, The Netherlands. ${ }^{2}$ Animal Breeding and Genomics Centre, Animal Science Group, Lelystad, The Netherlands.}

\section{Authors' contributions}

AC analysed the data and wrote the manuscript. MPLC was involved in the discussion and conclusion of the manuscript.

\section{Competing interests}

The authors declare no competing interests.

Published: 27 May 2011

\section{References}

1. Grapes $L$, Dekkers JCM, Rothschild MF, Fernando RL: Comparing linkage disequilibrium-based methods for fine mapping quantitative trait loci. Genetics 2004, 166:1561-1570.

2. Meuwissen THE, Hayes BJ, Goddard ME: Prediction of total genetic value using genome-wide dense marker maps. Genetics 2001, 157:1819-1829.

3. Bjornstad A, Westad F, Martens H: Analysis of genetic marker-phenotype relationships by jack-knifed partial least squares regression (PLSR). Hereditas 2004, 141(2):149-165.

4. Gilmour AR, Cullis BR, Welham SJ, Thompson R: ASREML Reference Manual. 2. NSW Agriculture, Locked Bag, Orange, NSW 2800, Australia; 2002.

5. R Development Core Team: R: A Language and Environment for Statistical Computing. R Foundation for Statistical Computing, Vienna, Austria;3-900051-07-0 2009.

6. Geladi P: Partial Least Squares Regression: a tutorial. Analytica Chimica Acta 1986, 185:1-17.

7. Wehrens R, Mevik BH: pls: Partial Least Squares Regression (PLSR) and Principal Component Regression (PCR). 2007, R package version 2.1-0.

8. Cleveland WS, Devlin SJ: Locally Weighted Regression: An Approach to Regression Analysis by Local Fitting. Journal of the American Statistical Association 1988, 83(403):596-610.

9. Chun $H$, Keles S: Sparse partial least squares regresssion for simultaneous dimension reduction and variable selection. $J$ R Stat Soc Series B Stat Methodol 2010, 72(1):3-25.

doi:10.1186/1753-6561-5-S3-S7

Cite this article as: Coster and Calus: Partial least square regression applied to the QTLMAS 2010 dataset. BMC Proceedings 2011 5(Suppl 3): S7. 